Respiratory and upper limb function as outcome measures in ambulant and non-ambulant subjects with Duchenne muscular dystrophy: a prospective multicentre study

V. Ricotti, V. Selby, D. Ridout, J. Domingos, V. Decostre, A. Mayhew, M. Eagle , J. Butler, M. Guglieri, M. Van der Holst, M. Jansen, J.J.G.M. Verschuuren, I.J.M. de Groot, E.H. Niks , L. Servais, V. Straub, T. Voit, J.Y. Hogrel, F. Muntoni

PII:

DOI: S0960-8966(18)31379-8

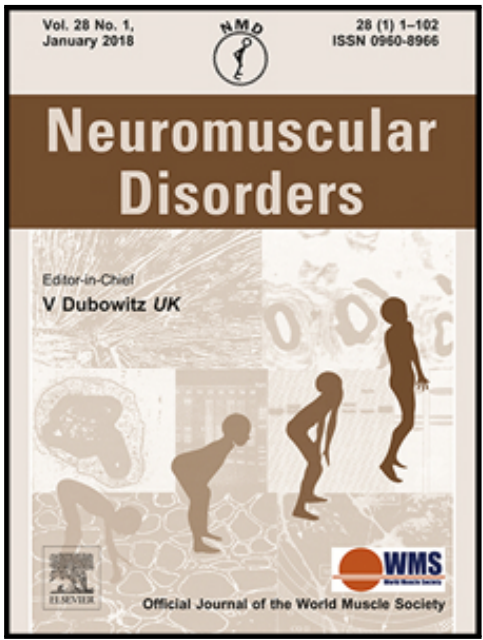

Reference: https://doi.org/10.1016/j.nmd.2019.02.002

To appear in: Neuromuscular Disorders

Received date: 17 December 2018

Revised date: $\quad 5$ February 2019

Accepted date:

8 February 2019

Please cite this article as: V. Ricotti, V. Selby, D. Ridout, J. Domingos, V. Decostre, A. Mayhew, M. Eagle, J. Butler, M. Guglieri, M. Van der Holst, M. Jansen, J.J.G.M. Verschuuren , I.J.M. de Groot, E.H. Niks, L. Servais, V. Straub, T. Voit, J.Y. Hogrel, F. Muntoni, Respiratory and upper limb function as outcome measures in ambulant and non-ambulant subjects with Duchenne muscular dystrophy: a prospective multicentre study , Neuromuscular Disorders (2019), doi: https://doi.org/10.1016/j.nmd.2019.02.002

This is a PDF file of an unedited manuscript that has been accepted for publication. As a service to our customers we are providing this early version of the manuscript. The manuscript will undergo copyediting, typesetting, and review of the resulting proof before it is published in its final form. Please note that during the production process errors may be discovered which could affect the content, and all legal disclaimers that apply to the journal pertain. 
Highlights

- This study contributes to the natural history of DMD, linking the ambulant and nonambulant phases

- Respiratory measurements, upper limb function, pinch and grip force are reported

- A composite score combining respiratory outcomes, upper limb function and strength is explored

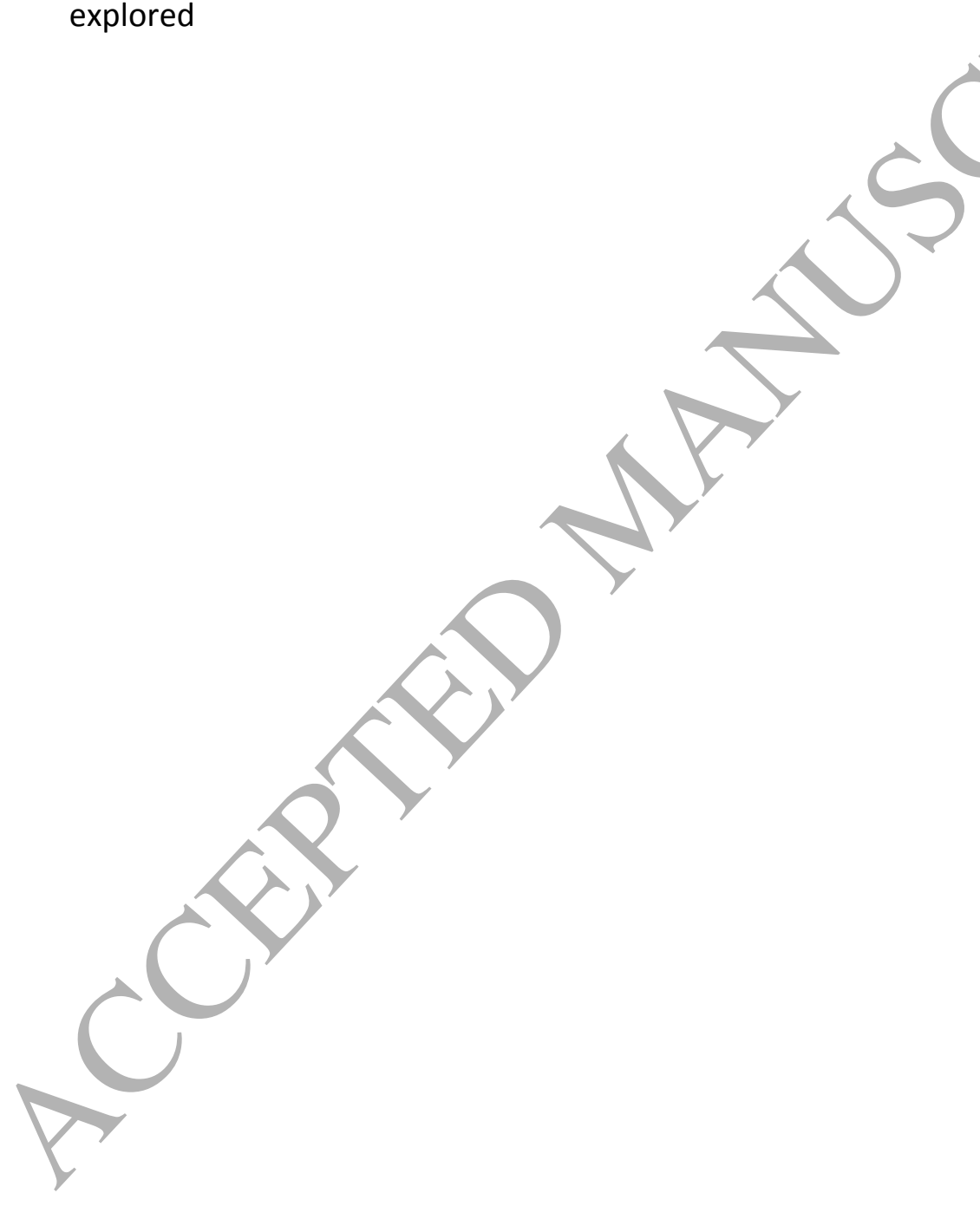




\section{Respiratory and upper limb function as outcome measures in ambulant and non-ambulant subjects with Duchenne muscular dystrophy: a prospective multicentre study.}

Ricotti, V. ${ }^{1,8^{*}}$, Selby, $\mathrm{V}^{1,2}$, Ridout, D. ${ }^{1,3}$; Domingos, $\mathrm{J}^{2{ }^{\dagger}}$, Decostre, V. ${ }^{4}$; Mayhew, A. ${ }^{5}$ Eagle, M. ${ }^{5}$; Butler, J. ${ }^{2}$ Guglieri, M. ${ }^{5}$; Van der Holst, M. ${ }^{6}$; Jansen, M. ${ }^{7}$; Verschuuren, J.J.G.M. ${ }^{6}$; de Groot, I.J.M. ${ }^{7}$; Niks, E. ${ }^{6}$;

Servais, L. ${ }^{4}$; Straub V ${ }^{5}$, Voit, T., ${ }^{1,2}$ Hogrel, J.Y. ${ }^{4}$; Muntoni, F. ${ }^{1,2^{*}}$

${ }^{1}$ NIHR Great Ormond Street Hospital Biomedical Research Centre, Great Ormond Street Institute of Child Health, University College London, \& Great Ormond Street Hospital Trust, London, UK

${ }^{2}$ Dubowitz Neuromuscular Centre, UCL Great Ormond Street Institute of Child Health \& Great Ormond Street Hospital Trust, London, UK

${ }^{3}$ Population, Policy and Practice Program, UCL Great Ormond Street Institute of Child Health, London, UK

${ }^{4}$ Institut de Myologie, Groupe Hospitalier Pitié Salpêtrière, Paris, France

${ }^{5}$ John Walton Muscular Dystrophy Research Centre, Newcastle University, Newcastle, UK

${ }^{6}$ Leiden University Medical Centre, Leiden, Netherlands

${ }^{7}$ Radboud University Nijmegen Medical Center, Donders Centre of Neuroscience, Department of Rehabilitation, Nijmegen, Netherlands

${ }^{8}$ Solid Biosciences, London, UK

${ }^{\dagger}$ Deceased

* Corresponding author 


\section{Corresponding authors contacts:}

\section{Professor Francesco Muntoni, FRCPCH, FMedSci}

Dubowitz Neuromuscular Centre and MRC Centre for Neuromuscular Diseases

NIHR Senior Investigator

UCL Great Ormond Street Institute of Child Health \& Great Ormond Street Hospital for Children (GOSH) Foundation Trust 30 Guilford Street, London WC1N 1EH, UK

Telephone: +442079052111 Fax: + 442079052832

Email: f.muntoni@ucl.ac.uk

Dr Valeria Ricotti, MRCPI, MRCPCH, MFPM, MD (Res)

Honorary Clinical Lecturer

NIHR Great Ormond Street Hospital Biomedical Research Centre, Great Ormond Street Institute of Child Health, University College London, \& Great Ormond Street Hospital Trust, London, UK

Telephone: +44207905 2211

Email: v.ricotti@ucl.ac.uk

\section{Abstract}

The field of translational research in Duchenne muscular dystrophy (DMD) has been

transformed in the last decade by a number of therapeutic targets, mostly studied in ambulant patients. A paucity of studies focus on measures that capture the non-ambulant stage of the disease, and the transition between the ambulant and non-ambulant phase.

In this prospective natural history study, we report the results of a comprehensive assessment of respiratory, upper limb function and upper limb muscle strength in a group of 89 DMD boys followed in 3 European countries, 81 receiving corticosteroids, spanning a wide age range (5-18 years) and functional abilities, from ambulant $(n=60)$ to non-ambulant $(n=29)$. 
Respiratory decline could be detected in the early ambulatory phase using Peak Expiratory Flow percentage predicted (PEF\%), despite glucocorticoid use (mean annual decline: $4.08,95 \% \mathrm{Cl}[-$ $7.44,-0.72], p=0.02$ in ambulant; 4.81, 95\%Cl [-6.79,-2.82], $p<0.001$ in non-ambulant). FVC\% captured disease progression in non-ambulant DMD subjects, with an annual loss of $5.47 \%$ (95\% Cl [-6.48,-4.45], p<0.001). Upper limb function measured with the Performance of Upper Limb (PUL 1.2) showed an annual loss of 4.13 points $(95 \% \mathrm{Cl}[-4.79,3.47], \mathrm{p}<0.001)$ in the nonambulant cohort. Measures of upper limb strength (MyoGrip and MyoRinch) showed a continuous decline independent of the ambulatory status, when reported as percentage predicted (grip force $-5.51 \%, 95 \% \mathrm{Cl}[-6.54,-4.48], \mathrm{p}<0.001$ in ambulant and a slower decline 2.86\%; $95 \% \mathrm{Cl}-3.29,-2.43, \mathrm{p}<0.001$, in non-ambulant; pinch force: $-2.66 \%, 95 \% \mathrm{Cl}[-3.82,-1.51]$, $p<0.001$ in ambulant and $-2.23 \%, 95 \% \mathrm{Cl}[-2.92,-1.53], p<0.001$ in non-ambulant).

Furthermore, we also explored the novel concept of a composite endpoint by combining respiratory, upper limb function and force domains: we were able to identify clear clinical progression in patients in whom an isolated measurement of only one of these domains failed to appreciate the yearly change. Our study contributes to the field of natural history of DMD, linking the ambulant and non-ambulant phases of the disease, and suggests that composite scores should be explored further.

\section{Introduction}

Duchenne muscular dystrophy (DMD) is a rare, X-linked neuromuscular disorder with an estimated incidence of approximately 1 in 3.500 to 1 in 5.000 live male births. [1-3] DMD is 
caused by mutations in the dystrophin gene $(D M D)$ that lead to an absence or near-absence of dystrophin, a protein essential for muscle cell integrity.[4] The profound deficiency of dystrophin seen in DMD results in progressive muscle degeneration and loss of function, culminating in premature death, typically by age 30.[5] DMD usually presents in early childhood with motor difficulties including delayed motor milestones, frequent falls, easy fatigability, as well as calf muscle hypertrophy. Mobility continues to decline over the course of the disease, with loss of ambulation by the early teen years and subsequent deterioration in upper extremity function to the point that patients are unable to perform even the most basic selfcare tasks. Scoliosis can develop due to weakness of trunk muscles and often requires surgical correction to maintain respiratory capacity.[6] Absence of dystrophin in the heart leads to cardiomyopathy, and respiratory muscle decline ultimately results in dependence on assisted ventilation.[7] Death is usually caused by cardiac and/or respiratory failure and related complications. $[8,9]$ There is currently no cure for DMD. DMD patients require a variety of interventions including medication, physiotherapy, nutritional and psychosocial support, and orthopaedic, respiratory, and cardiac care. [10-12] The current mainstay of DMD therapy consists of oral glucocorticoids, which improve muscle strength and delay loss of ambulation, development of cardiomyopathy and the need for ventilatory support.[13] Deflazacort recently received Food and Drug Administration USA (FDA) approval.[14] Furthermore, new therapies to increase dystrophin production in small genetic subsets of DMD have recently become available: ataluren (conditional approval in EU); [15] eteplirsen (accelerated approval in US). [16] Finally, a number of adeno-associated virus (AAV) mediated micro-dystrophin gene transfer clinical trials are on-going. However, clinical trials target mostly ambulant patients and 
this is reflected in the choice of the primary outcomes (e.g. six-minute walking test, NorthStar ambulatory assessment, 4 stair climb) excluding the participation of patients who are about to lose or already lost ambulation. Among the few exceptions, the trials evaluating idebenone (NCT03603288, NCT01027884) [17-19], pamrevlumab (NCT02606136), allogenic cardiospheres (NCT03406780), and AVV-mediated gene therapy IGNITE-DMD (NCT03368742) included nonambulant patients.

The increased survival and prolonged functional capacity of DMD subjects are important incentives to target clinical trials also towards non-ambulant DMD patients; in addition capturing the transition phase between ambulant and non-ambulant patients with meaningful outcome measures is important, as the eventual loss of ambulation is a likely event even for the ambulant DMD patients receiving experimental therapies. Therefore, the success of those trials depends on the establishment of outcome measures that are reliable and sensitive to change in disease progression across the loss of ambulation. During the non-ambulant phase of the disease, natural history studies on upper limb and respiratory function are critical to provide relevant information, as highlighted in recent literature regarding pulmonary endpoints in DMD. $[9,20,21]$ Furthermore, in response to the demand for monitoring motor function in the older subjects, the Performance of the Upper Limb (PUL), a functional scale dedicated to evaluating upper extremities in DMD has been developed,[22] (Mayhew et al. under review) necessitating further evaluation in other cohorts of patients.[23-26]

The aim of this prospective, longitudinal, multicentre natural history study was to provide novel information on outcome measures that can support drug development in DMD subjects 
irrespective of their ambulatory status. Respiratory function, upper limb function and strength measurements were included in the assessments. Furthermore, with a focus on the nonambulant cohort, the concept of a composite endpoint was explored by integrating the components of respiratory function, upper limb function and strength, with the goal to capture disease progression with a multi-component approach.

\section{Methods}

DMD subjects with a confirmed molecular and clinical diagnosis were recruited as part of a prospective, longitudinal, multicentre study across 5 centres in Europe (London and Newcastle, UK; Paris, France; Leiden and Nijmegen, The Netherlands), which aimed to assess the natural history of ambulant and non-ambulant patients with DMD. The key inclusion criteria for ambulant subjects were the following: age above 5 years old with a diagnosis of DMD documented by genetic testing (if a muscle biopsy was available, it had to contain less than $10 \%$ of revertant fibres) able to walk independently for at least 75 meters in 6 minutes at recruitment; receiving the standards of care for DMD as recommended by the DMD Care Considerations Working Group;[27] having a percentage predicted forced vital capacity (FVC) above $30 \%$. The key inclusion criteria for non-ambulant subjects were as follows: age between 5 and 18 years, loss of the ability to walk 10 meters without support; being capable of sitting upright in a wheelchair. Any subject with severe intellectual impairment that prevented cooperation during examination, symptomatic cardiac failure or anticipated surgery within 2 years from recruitment was excluded from the study. Subjects were assessed 6-monthly 
according to a shared protocol including among other measurements the Performance of Upper Limb (PUL version 1.2)[22, 24] and respiratory function (absolute and percentage predicted values for FVC and peak expiratory flow (PEF)) and strength measurement with the MyoGrip and MyoPinch dynamometers.[28] For strength measurements, the dominant side was assessed. All patients were given between two and five trials and the maximal value was recorded. For each muscle function tested, if the difference between the first two measurements was lower than $10 \%$ of the greater, the greater was accepted. If not, a subsequent measurement was made until two trials ranged within $10 \%$ (see Servais et al. 2013, for more details). The subjects were vigorously encouraged to produce their maximal voluntary effort. Grip and pinch maximal strength were expressed in $\mathrm{kg}$ and percentage of predicted values for age using predictive equations computed on a control population (internal database of the Institute of Myology, Paris and UCL Great Ormond Street Institute of Child Health \& Great Ormond Street Hospital Trust, London). Part of the data have been published for grip strength.[29] The respiratory function tests were performed by qualified respiratory physiologists or specialist neuromuscular physiotherapists according to the recommendations of the American Thoracic Society and the European Respiratory Society (ATS/ERS).[30] Each parameter was expressed as an absolute value and corresponding percentage predicted value. The absolute value was selected as the largest from 3 consecutives attempts at each visit. The percentage predicted value was determined using the relevant reference equations (best effort/predicted x100).[31, 32] For non-ambulant patients, ulnar length or arm span was used to derive height. Arm span was determined for each individual with arms extended laterally with palms facing forward, kept at shoulder height, from the tip of the middle (longest) finger 
of one side to the other recorded to the nearest $0.1 \mathrm{~cm}$ with a flexible, non-stretch tape. For the weakest patients with joint contractures, measurements were performed by adding the following segment lengths: right hand, forearm, arm, trunk width, left arm, forearm, hand. General demographics were also collected including date of birth, type of mutation and steroid treatment and regime. Steroid regime was defined as daily when taken every day, and intermittent when taken with different intervals (alternate days; periods of 10 days taking the medication followed by 10 days not taking it). Training by the same lead physiotherapist was provided to all clinical evaluators to ensure standardization of assessment procedures and scoring.

Ethics review boards at participating institutions approved the study protocol, consent and assent documents. Informed consent/assent was obtained for each participant prior to conducting the study. This study is registered with the Clinical Trial Gov website with the number: NCT02780492.

\section{Statistical methods}

Characteristics of the sample are presented as mean (SD), or frequency (percentage) unless otherwise stated. Considering ambulant and non-ambulant boys separately and using all available outcome data, we estimated the annual change for each of the outcomes using mixed effects regression models, accounting for the longitudinal data. All models were adjusted for steroid regimen and age at entry to the study. Boys who lost ambulation during the study were included in both sets of analyses and their age at the first visit after losing ambulation was used 
as baseline age in the non-ambulant models. Results are presented as mean annual change with $95 \%$ confidence intervals. In addition, as the outcomes are measured on different scales, we calculated standardised annual changes, using an internal standardisation for the ambulant cohort and for the non-ambulant cohort. The standardised annual change is a re-scaling of the annual change, it corresponds to the average annual change relative to the variability of the change between boys.

For the non-ambulant cohort we defined a composite score involving 3 outcomes, representing 3 different clinical aspects of function. For PUL total score we defined an annual decline in score during year 1 (between visit 1 and visit 3) of >=4 points, and an annual improvement in score of $>=4$ points as clinically meaningful. This was also based on previously reported data.[33] Similarly, for FVC \% we defined an annual change of more than $5 \%$ as meaningful [21] and for MyoGrip measurement of force, annual changes greater than 3\% were used. [34] Boys who experienced a deterioration for a particular function based on the criteria above were assigned a score of -1 . Vice-versa, where improvement was observed a score of +1 was assigned. Where no change was observed the boy was assumed to be stable for the function and given a score of zero. We calculated the composite score for each of the non-ambulant boys by summing these 3 scores and where data was not complete for year 1 for a particular boy we used the first available complete data in subsequent years. All analyses were conducted in Stata v15 [35] and a $P$ value $<0.05$ was considered statistically significant. 


\section{Results}

In this prospective and on-going study a total of 89 boys were included with confirmed clinical and genetic diagnosis of DMD (Table 1). All mutations were predicted to lead to a DMD phenotype, the majority being out of frame $D M D$ gene deletions (see Table 1-appendix). At recruitment 60 boys were ambulant with a mean age of 7.9 years (ranges: 5 - 13.6 years). The majority of the ambulant DMD boys (40/60) were on daily glucocorticoids, 17 on intermittent and 3 had not started glucocorticoids yet. The remaining 29 boys were non-ambulant with a mean age of 14.2 (ranges: 8.4, 18 years), of which 24 were on glucocorticoids (15 on a daily regimen) and 5 had stopped glucocorticoid therapy after loss of independent ambulation. Boys were assessed every 6 months. The majority of subjects $(n=75,84 \%)$ had at least 3 visits, so 1 year follow-up (table1). Eleven subjects lost independent ambulation during follow-up.

In relation to respiratory function (Figure $1 \mathrm{~A}$ ), a significant decline in FVC \% predicted was observed in the non-ambulant population consisting of $5.47 \%(95 \% \mathrm{Cl}[-6.48,-4.45], p<0.001)$ annual decline, whilst no significant change was observed in the FVC \% of ambulant boys (1.92, 95\% Cl $[-0.30,4.14], p=0.09)$. A decline in PEF\% predicted was observed as early as in the ambulant stage of the disease and further into the non-ambulant stage with a PEF \% annual deterioration of $4.08 \%(95 \% \mathrm{Cl}[-7.44,-0.72], \mathrm{p}=0.02)$ and $4.81 \%(95 \% \mathrm{Cl}[-6.79,-2.82]$, $p<0.001$ ) respectively (Table 2 ). Upper limb function assessed by the PUL remained stable in the ambulant population, while in the non-ambulant patients an annual total loss of 4.13 PUL points $(95 \% \mathrm{Cl}[-4.79,-3.47], \mathrm{p}<0.001)$ and a loss of 0.97 point at the shoulder level $(95 \% \mathrm{Cl}[-$ 
1.25, -0.69], $\mathrm{p}<0.001)$ was observed (Table 2, Figure 1B). When expressed in absolute values (kg), the strength for both grip and pinch shows a significant improvement in ambulant patients, followed by a decrease in non-ambulant patients. However, when expressed in percentage of predicted values, measurements of upper limb force revealed a steady decline across age and ambulatory status with an annual loss of grip force of $5.51 \%$ predicted $(95 \% \mathrm{Cl}[-$ $6.54,-4.48], \mathrm{p}<0.001)$ and $2.86 \%$ predicted $(95 \% \mathrm{Cl}[-3.29,-2.43], \mathrm{p}<0.001)$ in the ambulant and non-ambulant DMD respectively, and a decline in pinch force of $2.66 \%$ (95\% Cl $[-3.82,-1.51]$, $\mathrm{p}<0.001)$ in ambulant and $2.23 \%(95 \% \mathrm{Cl}[-2.92,-1.53], \mathrm{p}<0.001)$ in non-ambulant subjects (Table 2, Figure 1C).Table 1 reports the annual changes in ambulant and non-ambulant subjects adjusted for age and glucocorticoids use at baseline. In the supplemental material (appendixtable 2), we report standardised annual change from ambulant and non-ambulant subjects.

Furthermore, focusing on the non-ambulant cohort alone, we explored the concept of a composite score by combining the FCV\% (change of $>=5 \%$ ), PUL total score (change of $>=4$ points) and the MyoGrip measurement of force (change of $>=3 \%$ ). Out of 28 non-ambulant subjects who had all the assessments done, 21 showed a global decline on the composite score, which might not have been captured by assessing one parameter alone (i.e. 12 subjects declined in respect to the PUL, 13 in respect to FVC\% and 14 in respect to grip force). Only one boy showed a decline in all the three domains: respiratory function, upper limb function and force. Four subjects $(12,14,24$ and 26$)$ showed decline in one domain and improvement in another resulting in a composite score that was indicative of stable disease. Two subjects remained stable across all the three domains. 


\section{Discussion}

In our multicentre, prospective, longitudinal natural history study, respiratory function, upper limb function and strength were assessed in a cohort of 89 ambulant and non-ambulant DMD subjects treated according to the international standards of care and evaluated with a standardised protocol by trained physiotherapists. The progression of the disease was highlighted across the ambulatory stages of the disease with a particular focus on those outcome measures that are independent from ambulation. We observed an annual loss FVC \% predicted of $5.47(95 \% \mathrm{Cl}-6.48,-4.45, p<0.001)$ in the non-ambulant subjects ( $n=29)$, which is also in line with what has been previously reported in the literature, [17, 36-38] while this measure did not decline in the ambulant population ( $n=60)$. In accordance with recent observations $[9,21]$ we observed a deterioration in PEF\% predicted in young ambulant children with an annual decline of $4.08 \mathrm{PEF} \%(95 \% \mathrm{Cl}-7.44,-0.72, \mathrm{p}=0.02)$, reflecting that maximal expiratory muscle pressure required to perform this assessment is impaired already in this young DMD population. In the non-ambulatory cohort the annual decline of PEF \% predicted was $4.81 \%(95 \% \mathrm{Cl}-6.79,-2.82, \mathrm{p}<0.001)$, hence a very similar annual decline as in the ambulant patients. Our data are in keeping with the only other natural history study that specifically explored this outcome in which a similar rate of yearly decline (5\%) was reported.[37] Percentage predicted PEF was the primary endpoint in clinical trials testing idebenone [39]. The phase 3 clinical trial reported higher rates of annual decline in the placebo group $(8.84 \%$ per year) compared with Mayer et al. and our findings.[37] However, in contrast with the patients 
in the natural history studies including ours, the ones in the clinical trial were not treated with glucocorticoids. [39]. Newer evidence from the CINRG natural history study indicates that steroids are capable of delaying the onset of respiratory force decline but do not alter the slope of decline once this has started. [21] Our data are consistent with the observations from the CINRG natural history study, confirming the similar course of disease in DMD between our European and the US studies.

Upper limb function evaluated by the PUL (v1.2) appears to be a more sensitive measure of disease progression in the non-ambulant cohorts than in the ambulant, principally because of a ceiling effect in the ambulant group. The total score showed an annual decline of 4.13 points $(95 \% \mathrm{Cl}-4.79,-3.47, \mathrm{p}<0.001)$, and a loss of performance at shoulder level of 0.97 scores $(95 \%$ $\mathrm{Cl}-1.25,-0.69, \mathrm{p}<0.001)$ in the non-ambulant population. In the ambulant population, the shoulder sub-domain detected a mild decline over the course of the year, which however was not statistically significant, but would be in line with the proximal to distal progression of the disease. These PUL findings corroborate the results observed in DMD populations followed at other European sites (Mayhew et al. under review )[25].

When measuring distal upper limb strength with the MyoGrip and MyoPinch [28] a steady decline of the percentage predicted grip and pinch force were observed. Grip force showed a more rapid annual decline in ambulant $(-5.51 \%, 95 \% \mathrm{Cl}[-6.54,-4.48], \mathrm{p}<0.001)$ and a slower decline in non-ambulant boys $(-2.86 \%[95 \% \mathrm{Cl}-3.29,-2.43] \mathrm{p}<0.001)$ when compared to pinch force $(-2.66 \%, 95 \% \mathrm{Cl}[-3.82,-1.51], \mathrm{p}<0.001$ in ambulant and $-2.23 \%, 95 \% \mathrm{Cl}[-2.92,-1.53]$, $\mathrm{p}<0.001$ in non-ambulant boys). Similar trajectories have been observed in previous studies 
using the same dynamometers. [34, 40] Importantly, these results demonstrate that DMD children never reach a normal force capacity when compared to healthy children. DMD children peak at about $60 \%$ of normal total grip force predicted. Results also emphasize how the distal upper extremities loose strength during the early stages of the disease. Muscle strength expressed as percentage predicted seems to be a consistent clinical outcome measure, which, together with the respiratory function measures, bridges the early ambulatory stage of DMD towards the later non-ambulatory phase. Non-ambulant subjects lose less strength probably because they have less total strength to lose. However, when looking at relative declines (with respect to their remaining strength), they lose more than ambulant patients.

Finally, with the aim to capture progression in multiple domains of DMD in the non-ambulant phase, we explored the concept of a composite endpoint using a descriptive analytical approach. With a focus on the non-ambulant cohort $(n=28)$, the following parameters were selected on the basis of the observed annual decline, which is also in line with the literature: FVC\% (5\% change), PUL total score (4 points change) and grip strength ( $3 \%$ change).[33, 34,37$]$ Table 3 highlights the high individual variability in disease progression when individual domains are considered: with the exception of one boy (subject 23) who showed decline in the respiratory, upper limb function and force domains, all other subjects showed a more heterogeneous picture. However, using our exploratory composite score, decline was detected in $21 / 28$ subjects ( $75 \%)$ over the course of one year. It must be highlighted that 4 subjects showed decline in one domain and improvement in another (subjects $12,14,24,26$ ) resulting in a composite score that is indicative of stable disease. 
We are aware that care should be used when considering a composite score, as the different subdomains are likely to progress with different slopes and have different linearity, and in this context more work will be required to assess the validity of our proposed exploratory measure in the future. We defined the thresholds for improvement or decline for each subdomains in the composite score at the mean annual decline considered clinically significant for that parameter, but we have not defined the relationship of linearity for each of these outcome measures. In addition, the minimally clinically important difference (MCID) for these domains have yet to be established: in our exploratory composite endpoint we therefore have weighted all 3 domains equally, however DMD boys may experience that a decline in one domain is more impactful than in the others. Furthermore, given the small number of patients included and the exploratory nature of this analysis, these results need to be interpreted with caution. This exploratory composite score is also meant to spark debate on the multiple dimension of disease progression that is not captured by the current assessment tools, and that it could be potentially complemented with other progression disease biomarkers such MRI, which has previously been shown to progress in close correlation with force/function measurements. [40, 41]

An example of a composite score is the one used in a phase 3 study of the safety and efficacy of laronidase in the storage disorder Mucopolysaccharidosis I (MPS I) (NCT00146770) comparing placebo with treatment arm. In this study, a composite endpoint was used that summed up clinically significant changes across five efficacy variables (percent predicted normal FVC, 6minute walk test distance, shoulder flexion range of motion, apnoea-hypopnoea index, and visual acuity), providing a global response to the treatment. A similar approach could 
potentially be explored also in DMD, given the heterogeneity of this disease and its multisystemic manifestations across the population. Of course, such concept requires further evaluation in larger cohorts of patients, and assessment of the contribution of the changes across the entire range of the scales for each of the sub-domains.

In summary, our prospective study, for the first time, combines outcomes of respiratory, upper limb function and precise upper limb force dynamometry, across ambulatory and nonambulatory DMD subjects. On the other hand and lending support to the validity of our observations, the rate of decline of PEF\% predicted and FVC \% predicted in non-ambulant patients as well as in the late ambulant phase are in the same range as those independently observed in the CINRG natural history study, but extended further to capture younger DMD boys. [21] Our study has however limitations. The small number of subjects included in our study, can limit some sub-group analyses and generalization of results. Secondly, our cohort of patients does not reflect the full genotype spectrum of DMD, and it is known that genotype can influence functional capacities. [25, 42-45]. Finally, patients in our cohorts were on different glucocorticoid regimens, the potential impact of which could not be compared due to the small numbers.

We demonstrate that progressive motor and respiratory function decline are features of DMD in the ambulant and non-ambulant phase of the disease, albeit at different rates for the different parameters studied. These observations should allow for clinical study designs, which 
aim at slowing decline or even improving function in DMD across the ambulant and nonambulant phases of the disease.

\section{Acknowledgments:}

This study is funded by the Association Française contre les Myopathies (AFM)

CERTUS technology is acknowledged for hosting the database.

JD was funded by the MRC Centre for Neuromuscular Diseases. The support of the UCL MRC Neuromuscular Biobank and of the Muscular Dystrophy UK to the Dubowitz Neuromuscular Centre is also gratefully acknowledged.

This research was supported by the NIHR Great Ormond Street Hospital Biomedical Research Centre. The views expressed are those of the author(s) and not necessarily those of the NHS, the NIHR or the Department of Health.

We would like to thank the additional clinicians for data collection. Newcastle: Robert Muni Lofra, Meredith James, Dionne Moat, Jassi Sodhi. Leiden: Janneke van Egmond-van Dam.

The authors wish to pay a special tribute to Dr Joana Domingos who sadly passed away after a very short illness in January 2018. Joana was studying towards a PhD on outcome measures for DMD. We want in particular to acknowledge both her contribution to the clinical care of the DMD boys recruited into this study, and to the mining of the consortium data.

\section{Authors contributions:}

VR contributed to protocol development, set up of the database, patients' recruitment, data collection, data curation, data analysis, wrote the first draft of the manuscript and contributed to its revision. 
VS contributed to patients' recruitment, data curation and manuscript revision.

DR contributed to the data curation, data analysis and revision of the manuscript.

VD contributed to protocol development, patients' recruitment, data curation and manuscript revision

JD contributed to data collection and data curation.

AM contributed to protocol development, data collection, data curation and manuscript revision.

ME to protocol development and manuscript revision

JB contributed to data collection and data curation

MG contributed to data collection and manuscript revision

$\mathrm{MVdH}$ contributed to data collection and manuscript revision

VJJGM contributed to patient recruitment and manuscript revision

IJMdG contributed to patient recruitment and manuscript revision

EHN contributed to patient recruitment and manuscript revision

LS contributed to patient recruitment and manuscript revision

VS contributed to patient recruitment and manuscript revision

TV contributed to protocol development and manuscript revision

JYH contributed to protocol development, data analysis, data curation and manuscript revision

FM obtained the funding, contributed to protocol development, patient recruitment and manuscript revision. 


\section{Competing interest's disclosure}

VR was a Solid Biosciences employee at the time of writing this manuscript.

AM has participated in SAB meetings for Summit, PTC and Biogen and performs Consultancy work (training physiotherapists) for: Roche, Pfizer, PTC, Summit, Sarepta, Santhera, Italfarmaco, Amicus, Biogen and Avexis.

ME is managing director of Atom International Limited.

MvdH performs Consultancy work (training physiotherapists) for: Roche, PTC, Sarepta, Santhera, Italfarmaco, Amicus and MNK

EHN reports grants from Spieren voor Spieren, Duchenne Parent Project, ZonMW and AFM, and was local PI in studies conducted by BioMarin, GSK, Lilly, Santhera, Italfarmaco, Roche, and WAVE Life Sciences outside the submitted work. He also reports consultancies for BioMarin, Summit and WAVE. All reimbursements were received by the LUMC. No personal financial benefits were received.

IdG has participated in consultancy and educational meetings of PTC and Sarepta. Radboudumc is participating in clinical trials from Italfarmaco, Santhera and Roche (IdG PI).

JJGMV has been involved in Duchenne trials that are sponsored by Biomarin/Prosensa, GSK, Santhera or Lilly, in two European FP7 project on Duchenne muscular dystrophy (BIOIMAGE NMD, \#602485, and SCOPE DMD, \#60157), and a natural history study supported by AFM. All reimbursements were received by the LUMC, JJGMV had no personal financial benefit.

LS is part of the SAB of Sarepta, Santhera, of the steering commitee of Roche. LS has consultancy running for Roche and Biophytis. LS is PI in Sarepta studies, Wave, Santhera and Givinostat. 
FM is a member of the Rare Disease Scientific Advisory Group for Pfizer, and has participated to SAB meetings for PTC, Sarepta, Wave Therapeutics and Summit. UCL and Great Ormond Street Hospital are recipient of grants from Pfizer, Italfarmaco, Wave, Sarepta and Summit regarding clinical trials (Muntoni PI).

TV is an SAB member of Constant Pharma and Metriopharm; he served as consultant for: Audentes, BioLeaders, Biophytis, Capricor, DebioPharm, Fibrogen, Italfarmaco, Lysogene, Santhera, Sarepta, Servier, Solid, and Summit. He acted as a PI for Prosensa, GSK, Sarepta, PTC, Santhera.

JYH is a co-inventor of the MyoGrip and MyoPinch devices.

All the other authors have no conflicts to declare.

\section{References}

[1] Jean K. Mah LK, Jonathan Dykeman, Lundy Day, Tamara Pringsheim, Nathalie Jette. A systematic review and meta-analysis of the epidemiology of Duchenne and Becker muscular dystrophy. Neuromuscular Disorders 2014;24:10.

[2] Mendell JR, Shilling C, Leslie ND, et al. Evidence-based path to newborn screening for Duchenne muscular dystrophy. Ann Neurol 2012;71:304-13.

[3] Ellis JA, Vroom E, Muntoni F. 195th ENMC International Workshop: Newborn screening for Duchenne muscular dystrophy 14-16th December, 2012, Naarden, The Netherlands. Neuromuscul Disord 2013;23:682-9.

[4] Blake DJ, Weir A, Newey SE, Davies KE. Function and genetics of dystrophin and dystrophin-related proteins in muscle. Physiol Rev 2002;82:291-329.

[5] Moxley RT, 3rd,Pandya S, Ciafaloni E, Fox DJ, Campbell K. Change in natural history of Duchenne muscular dystrophy with long-term corticosteroid treatment: implications for management. J Child Neurol 2010;25:1116-29.

[6] Apkon SD, Alman B, Birnkrant DJ, et al. Orthopedic and Surgical Management of the Patient With Duchenne Muscular Dystrophy. Pediatrics 2018;142:S82-S89.

[7] Eagle M, Baudouin SV, Chandler C, Giddings DR, Bullock R, Bushby K. Survival in Duchenne muscular dystrophy: improvements in life expectancy since 1967 and the impact of home nocturnal ventilation. Neuromuscul Disord 2002;12:926-9.

[8] Kamdar F, Garry DJ. Dystrophin-Deficient Cardiomyopathy. J Am Coll Cardiol 2016;67:2533-46. 
[9] LoMauro A, D'Angelo MG, Aliverti A. Assessment and management of respiratory function in patients with Duchenne muscular dystrophy: current and emerging options. Ther Clin Risk Manag 2015;11:1475-88.

[10] Birnkrant DJ, Bushby K, Bann CM, et al. Diagnosis and management of Duchenne muscular dystrophy, part 2: respiratory, cardiac, bone health, and orthopaedic management. Lancet Neurol 2018;17:347-61.

[11] Birnkrant DJ, Bushby K, Bann CM, et al. Diagnosis and management of Duchenne muscular dystrophy, part 1: diagnosis, and neuromuscular, rehabilitation, endocrine, and gastrointestinal and nutritional management. Lancet Neurol 2018;17:251-67.

[12] Birnkrant DJ, Bushby $\mathrm{K}$, Bann CM, et al. Diagnosis and management of Duchenne muscular dystrophy, part 3: primary care, emergency management, psychosocial care, and transitions of care across the lifespan. Lancet Neurol 2018;17:445-55,

[13] Matthews E, Brassington R, Kuntzer T, Jichi F, Manzur AY. Corticosteroids for the treatment of Duchenne muscular dystrophy. Cochrane Database Syst Rev 2016:CD003725.

[14] Administration USFaD, FDA approves drug to treat Duchenne muscular dystrophy. 2017.

[15] Excellence N-NIfHaC. Ataluren for treating Duchenne muscular dystrophy with a nonsense mutation in the dystrophin gene. In: Excellence N-N I f H a C, 2016.

[16] Administration F-UFD, FDA grants accelerated approval to first drug for Duchenne muscular dystrophy, FDA, Editor. 2016.

[17] Buyse GM, Goemans N, van den Hauwe M, et al. Idebenone as a novel, therapeutic approach for Duchenne muscular dystrophy: results from a 12 month, double-blind, randomized placebo-controlled trial. Neuromuscul Disord 2011;21:396-405.

[18] Buyse GM, Goemans N, van den Hauwe M, Meier T. Effects of glucocorticoids and idebenone on respiratory function in patients with duchenne muscular dystrophy. Pediatr Pulmonol 2013;48:912-20.

[19] Buyse GM, Voit T, Schara $U$, et al. Efficacy of idebenone on respiratory function in patients with Duchenne muscular dystrophy not using glucocorticoids (DELOS): a double-blind randomised placebo-controlled phase 3 trial. Lancet 2015;385:1748-57.

[20] Finder J, Mayer OH, Sheehan D, et al. Pulmonary Endpoints in Duchenne Muscular Dystrophy. A Workshop Summary. Am J Respir Crit Care Med 2017;196:512-19.

[21] McDonald CM, Gordish-Dressman H, Henricson EK, et al. Longitudinal pulmonary function testing outcome measures in Duchenne muscular dystrophy: Long-term natural history with/and without glucocorticoids. Neuromuscul Disord 2018.

[22] Mayhew A, Mazzone ES, Eagle M, et al. Development of the Performance of the Upper Limb module for Duchenne muscular dystrophy. Dev Med Child Neurol 2013;55:103845.

[23] Pane M, Fanelli L, Mazzone ES, et al. Benefits of glucocorticoids in non-ambulant boys/men with Duchenne muscular dystrophy: A multicentric longitudinal study using the Performance of Upper Limb test. Neuromuscul Disord 2015;25:749-53.

[24] Pane M, Mazzone ES, Fanelli L, et al. Reliability of the Performance of Upper Limb assessment in Duchenne muscular dystrophy. Neuromuscul Disord 2014;24:201-6.

[25] Pane M, Mazzone ES, Sormani MP, et al. 6 Minute walk test in Duchenne MD patients with different mutations: 12 month changes. PLoS One 2014;9:e83400. 
[26] Han JJ, de Bie E, Nicorici A, et al. Reachable workspace and performance of upper limb (PUL) in duchenne muscular dystrophy. Muscle Nerve 2016;53:545-54.

[27] Bushby K, Finkel R, Birnkrant DJ, et al. Diagnosis and management of Duchenne muscular dystrophy, part 1: diagnosis, and pharmacological and psychosocial management. Lancet Neurol 2010;9:77-93.

[28] Servais L, Deconinck N, Moraux A, et al. Innovative methods to assess upper limb strength and function in non-ambulant Duchenne patients. Neuromuscul Disord 2013;23:139-48.

[29] Hogrel JY. Grip strength measured by high precision dynamometry in healthy subjects from 5 to 80 years. BMC Musculoskelet Disord 2015;16:139.

[30] Miller MR, Hankinson J, Brusasco V, et al. Standardisation of spirometry. Eur Respir J 2005;26:319-38.

[31] Domenech-Clar R, Lopez-Andreu JA, Compte-Torrero L, et al. Maximal static respiratory pressures in children and adolescents. Pediatr Pulmonol 2003;35:126-32.

[32] Quanjer PH, Stanojevic S, Cole TJ, et al. Multi-ethnic reference values for spirometry for the 3-95-yr age range: the global lung function 2012 equations. Eur Respir J 2012;40:1324-43.

[33] Pane M, Coratti G, Brogna C, et al. Upper limb function in Duchenne muscular dystrophy: 24 month longitudinal data. PLoS One 2018;13:e0199223.

[34] Seferian AM, Moraux A, Annoussamy M, et al. Upper limb strength and function changes during a one-year follow-up in non-ambulant patients with Duchenne Muscular Dystrophy: an observational multicenter trial. PLoS One 2015;10:e0113999.

[35] StataCorp., Stata Statistical Software. 2017, College Station, TX: StataCorp LLC.

[36] Khirani S, Ramirez A, Aubertin G, et al. Respiratory muscle decline in Duchenne muscular dystrophy. Pediatr Pulmonol 2014;49:473-81.

[37] Mayer $\mathrm{OH}$, Finkel RS, Rummey $\mathrm{C}$, et al. Characterization of pulmonary function in Duchenne Muscular Dystrophy. Pediatr Pulmonol 2015;50:487-94.

[38] Connolly AM, Florence JM, Zaidman CM, et al. Clinical trial readiness in non-ambulatory boys and men with duchenne muscular dystrophy: MDA-DMD network follow-up. Muscle Nerve 2016;54;681-9.

[39] Buyse GM, Voit T, Schara U, et al. Efficacy of idebenone on respiratory function in patients with Duchenne muscular dystrophy not using glucocorticoids (DELOS): a double-blind randomised placebo-controlled phase 3 trial. Lancet 2015;385:1748-1757.

[40] Hogrel JY, Wary C, Moraux A, et al. Longitudinal functional and NMR assessment of upper limbs in Duchenne muscular dystrophy. Neurology 2016;86:1022-30.

[41] Ricotti V, Evans MR, Sinclair CD, et al. Upper Limb Evaluation in Duchenne Muscular Dystrophy: Fat-Water Quantification by MRI, Muscle Force and Function Define Endpoints for Clinical Trials. PLoS One 2016;11:e0162542.

[42] Ricotti V, Ridout DA, Pane M, et al. The NorthStar Ambulatory Assessment in Duchenne muscular dystrophy: considerations for the design of clinical trials. J Neurol Neurosurg Psychiatry 2016;87:149-55.

[43] Bello L, Morgenroth LP, Gordish-Dressman H, et al. DMD genotypes and loss of ambulation in the CINRG Duchenne Natural History Study. Neurology 2016;87:401-9. 
[44] van den Bergen JC, Ginjaar HB, Niks EH, Aartsma-Rus A, Verschuuren JJ. Prolonged Ambulation in Duchenne Patients with a Mutation Amenable to Exon 44 Skipping. J Neuromuscul Dis 2014;1:91-4.

[45] Servais L, Montus M, Guiner CL, et al. Non-Ambulant Duchenne Patients Theoretically Treatable by Exon 53 Skipping have Severe Phenotype. J Neuromuscul Dis 2015;2:26979.

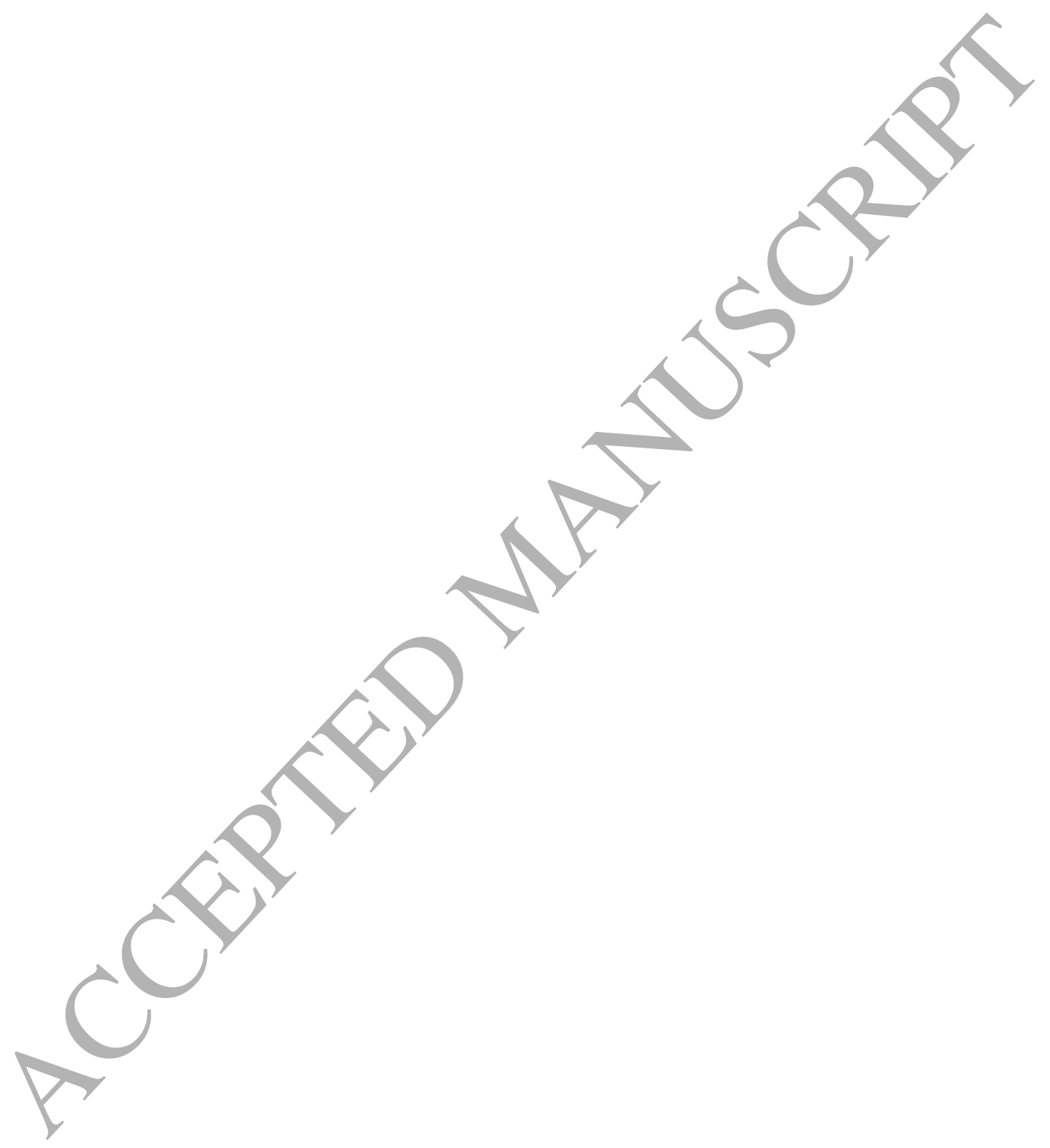




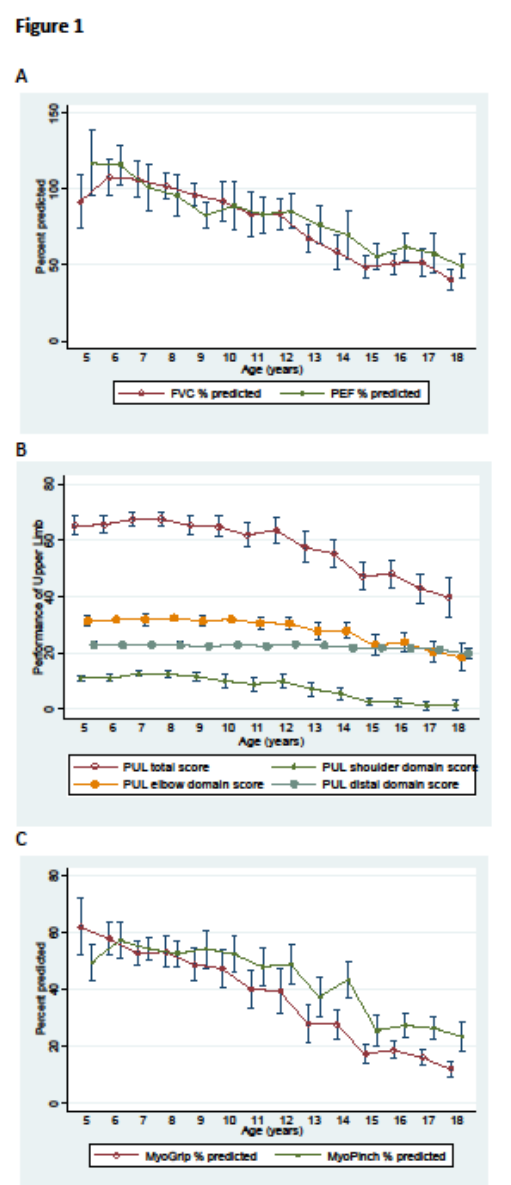

Figure 1. Mean and $95 \% \mathrm{Cl}$ in ambulant and non-ambulant DMD boys for $(\mathrm{A})$ respiratory function (FVC\% and PEF\%) in relation to age (B) Performance of the upper limb functional scale: total score and sub-domains in relation to age (C) MyoGrip and MyoPinch in \% of predicted values for age. Note: maximum PUL 1.2 total score $=74$, maximum shoulder domain score $=16$; maximum elbow domain score $=34 ;$ maximum distal domain score 24 . 
Table 1. General characteristics of subjects at their initial assessment

$F V C=$ force vital capacity; $P E F=$ peak expiratory force; $P U L=$ performance of the upper limb

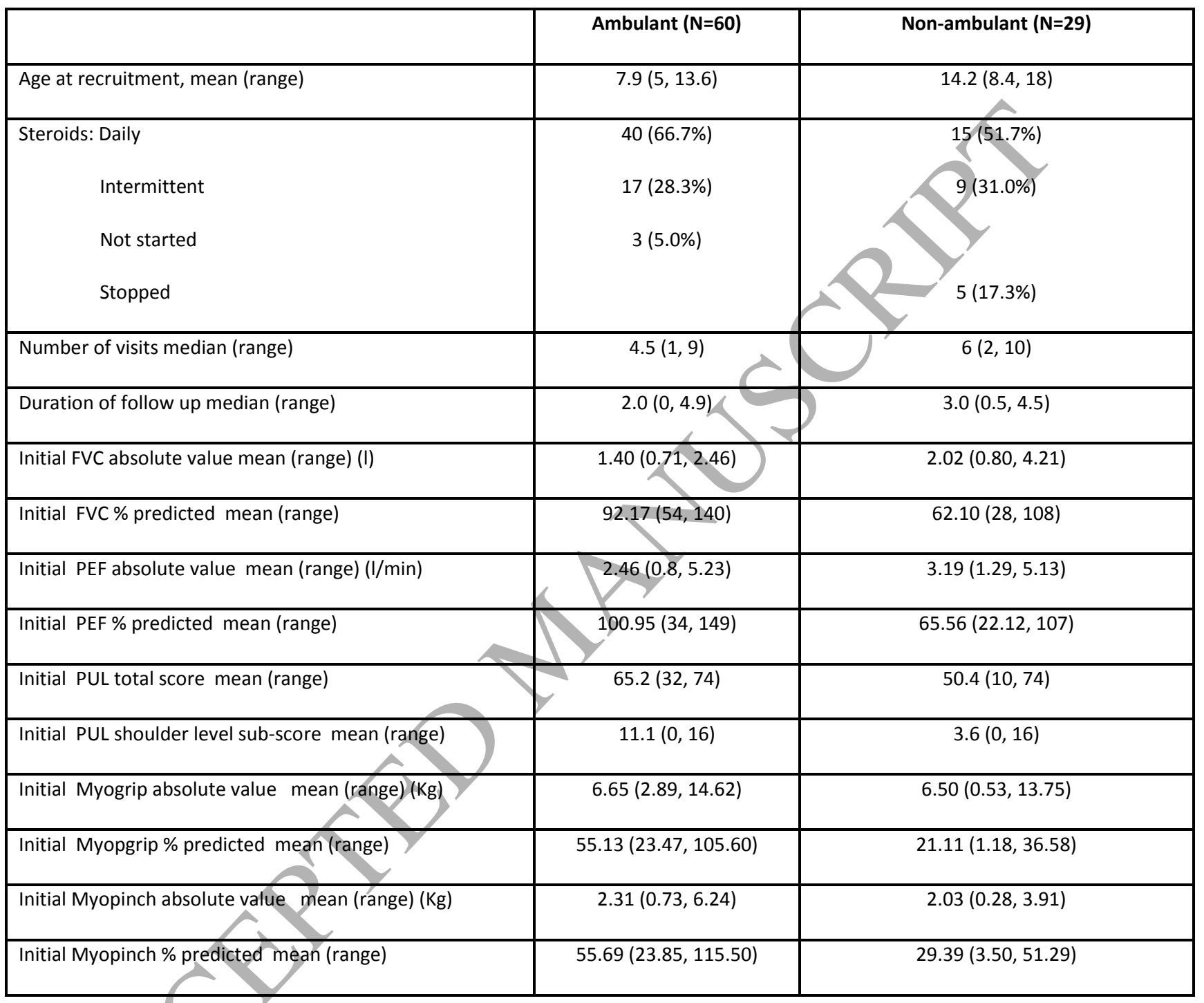


Table 2. Estimated annual changes from baseline for respiratory and upper limb

measurements

$F V C=$ force vital capacity; $P E F=$ peak expiratory force; $P U L=$ performance of the upper limb

\begin{tabular}{|c|c|c|}
\hline & $\begin{array}{c}\text { AMBULANT }(n=60) \\
\text { Mean change }(95 \% \mathrm{Cl}) \\
\text { p- value }\end{array}$ & $\begin{array}{c}\text { NON-AMBULANT }(n=29) \\
\text { Mean change }(95 \% \mathrm{Cl}) \\
\text { p-value }\end{array}$ \\
\hline FVC absolute value (I) & $\begin{array}{c}0.14(0.11,0.17) \\
<0.001\end{array}$ & $\begin{array}{c}-0.06(-0.10,-0.02) \\
<0.01\end{array}$ \\
\hline FVC \% predicted & $\begin{array}{c}1.92(-0.30,4.14) \\
0.09\end{array}$ & $\begin{array}{c}-5.47(-6.48,-4.45) \\
<0.001\end{array}$ \\
\hline PEF absolute value (I/min) & $\begin{array}{c}0.45(0.34,0.56) \\
<0.001\end{array}$ & $\begin{array}{c}0.24(0.10,0.37) \\
<0.001\end{array}$ \\
\hline PEF \% predicted & $\begin{array}{c}-4.08(-7.44,-0.72) \\
0.02\end{array}$ & $\begin{array}{c}-4.81(-6.79,-2.82) \\
<0.001\end{array}$ \\
\hline PUL total score & $\begin{array}{c}0.36(-0.62,1.34) \\
0.48\end{array}$ & $\begin{array}{c}-4.13(-4.79,-3.47) \\
<0.001\end{array}$ \\
\hline PUL shoulder level sub-score & $\begin{array}{c}-0.13(-0.62,0.36) \\
0.61\end{array}$ & $\begin{array}{c}-0.97(-1.25,-0.69) \\
<0.001\end{array}$ \\
\hline Myogrip absolute value (Kg) & 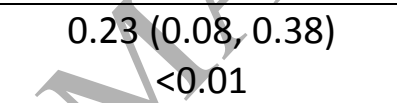 & $\begin{array}{c}-0.39(-0.50,-0.29) \\
<0.001\end{array}$ \\
\hline Myopgrip \% predicted & $\begin{array}{c}-5.51(-6.54,-4.48) \\
<0.001\end{array}$ & $\begin{array}{c}-2.86(-3.29,-2.43) \\
<0.001\end{array}$ \\
\hline Myopinch absolute value (Kg) & $\begin{array}{c}0.09(0.04,0.14) \\
<0.01\end{array}$ & $\begin{array}{c}-0.08(-0.13,-0.03) \\
<0.01\end{array}$ \\
\hline Myopinch \% predicted & $\begin{array}{c}-2.66(-3.82,-1.51) \\
<0.001\end{array}$ & $\begin{array}{c}-2.23(-2.92,-1.53) \\
<0.001\end{array}$ \\
\hline
\end{tabular}


Table 3. Composite endpoint in non-ambulant DMD integrating PUL total score , FVC\% predicted and MyoGrip.

A clinically meaningful change for the PUL total score $=4$ points, for the FVC $\%=5 \%$ and for the MyoGrip $=3 \%$. A decline was captured as -1 , unchanged measurements as 0 and improvement as 1 .

\begin{tabular}{|c|c|c|c|}
\hline Subject & $\begin{array}{l}\text { PUL } \\
\text { total }\end{array}$ & FVC\% & MyoGrip\% \\
\hline 1 & -1 & -1 & -1 \\
\hline 2 & -1 & -1 & 0 \\
\hline 3 & -1 & -1 & 0 \\
\hline 4 & -1 & -1 & 0 \\
\hline 5 & -1 & 0 & -1 \\
\hline 6 & -1 & 0 & -1 \\
\hline 7 & -1 & 0 & 0 \\
\hline 8 & -1 & 0 & 0 \\
\hline 9 & -1 & 0 & 0 \\
\hline 10 & -1 & 1 & -1 \\
\hline 11 & -1 & 1 & 0 \\
\hline 12 & -1 & 1 & 0 \\
\hline 13 & 0 & -1 & -1 \\
\hline 14 & 0 & -1 & -1 \\
\hline 15 & 0 & -1 & -1 \\
\hline 16 & 0 & -1 & -1 \\
\hline 17 & 0 & -1 & 0 \\
\hline 18 & 0 & -1 & 0 \\
\hline 19 & 0 & -1 & 0 \\
\hline 20 & 0 & 0 & -1 \\
\hline 21 & 0 & 0 & -1 \\
\hline 22 & 0 & 0 & 0 \\
\hline 23 & 0 & 0 & 0 \\
\hline 24 & 0 & 1 & 0 \\
\hline 25 & 1 & -1 & -1 \\
\hline 26 & 1 & -1 & -1 \\
\hline 27 & 1 & 0 & -1 \\
\hline 28 & 1 & 0 & -1 \\
\hline
\end{tabular}

